\title{
Chronic Pancreatitis - A Slave for Many Masters
}

\section{KS Santhosh Anand*}

Consultant, Surgical Gastroenterology, Dharan Super Speciality Hospital, Salem, India.

*Corresponding Author: KS Santhosh Anand, Consultant, Surgical Gastroenterology, Dharan Super Speciality Hospital, Salem, India.

Received: September 19, 2019; Published: October 01, 2019

DOI: $10.31080 /$ ASGIS.2019.02.0085

Pancreas has always been an intimidating organ. A medical student recognises it as a nightmare as initial medical school teaches "not to touch but just to see". It threatens trauma surgeons particularly during blunt abdominal trauma being diagnosed delayed. Alcohol and gall stones bite it in one fifth of patients causing severe acute pancreatitis, where destiny is decided when the process kicks start, giving less options for both patients and doctor in the due course. Adenocarcinoma of head region presents early, amenable for surgery, yet survival and prognosis is not great.

With all these threat and pessimism, there is one disease of pancreas for which physicians and surgeons claim their copyrights. Chronic pancreatitis has a spectrum of manifestations. It punishes the patient with exhilarating pain to begin with. However as the disease progresses, it disguises itself becoming patient friendly causing less and less pain. But poor patient ultimately realises that it has cursed him with brittle diabetes and steatorrhoea making him crippled. It is in between the ends of this spectrum, patient knocks hospital door multiple times, sometimes to a physician and at times to a surgeon.

Unlike other diseases of pancreas, chronic pancreatitis had been and is continuing to be in gray area may it be its cause, its course or its management. Physician pens down few drugs for his pain and enhanced diet absorption. Intervention gastroenterologist inserts his endoscope removes the stone from its duct and assures near cure. While surgeon opens the duct, removes all stones and cores head parenchyma and claims durable pain relief. With increasing research, many studies prove superiority of medicine, surgery and intervention over one another.

The confusion is not only created by diversity in management among medical professionals, but also by half baked details easily available in search engines to patients. Doctor shopping perplexes him and internet screws him telling he can end up with cancer. The latter can be still true with increased incidence in patients with chronic pancreatitis, but one should remember it does not occur in all.

The quality of a doctor depends on his holistic approach with patient. One should not just assess disease extent, but also should gauge social and logistic background of patient and tailor the treatment according to patient. Unfortunately, the latter two things are not taught formally during medical or surgical curriculum and have to be gained by experience only.

Prevention is always better than cure. Alcohol being the most common cause for chronic pancreatitis, curbing its use will benefit patient from many other menaces. As responsible health care professionals, our duty should start from community prevention programmes. With adequate scientific knowledge, we should appraise patient's need and tackle it accordingly.

A middle aged reformed alcoholic stepped in to my consultation room with reports saying he had mass in head of pancreas with jaundice in the background of chronic pancreatitis. He was stented to relieve jaundice and was told to be in supportive care as his mass is neither resectable nor to expect good response from chemotherapy. I consoled him with empathy saying it could be inflammatory mass too, but cannot rule out cancer. Same patient met me after 3 months healthy and enthusiastic. His mass by now had disappeared and he grinned as if he had escaped from death.

Sometimes masterly inactivity happens to be the best treatment. With so many gray areas in chronic pancreatitis, this ball will stay in courts of physician, endoscopist and surgeon at different times, but it will not become slave of anyone alone. Many things are yet to be understood and interpreted properly. Till then, let's provide the best possible treatment gauged to each and every patient individually.

Volume 2 Issue 9 November 2019

(C) All rights are reserved by KS Santhosh Anand. 